\title{
HIGHER-DIMENSIONAL SHIFT EQUIVALENCE AND STRONG SHIFT EQUIVALENCE ARE THE SAME OVER THE INTEGERS
}

\author{
J. B. WAGONER
}

(Communicated by Frederick R. Cohen)

\begin{abstract}
Let $R S(\Lambda)$ and $S(\Lambda)$ denote, respectively, the spaces of strong shift equivalences and shift equivalences over a subset $\Lambda$ of a ring which is closed under addition and multiplication. For example, let $\Lambda$ be the integers $Z$ or the nonnegative integers $Z^{+}$. For any principal ideal domain $\Lambda$, we prove that the continuous map $R S(\Lambda) \rightarrow S(\Lambda)$ is a homotopy equivalence. The methods also show that any inert automorphism, i.e, an element in the kernel of $\pi_{1}\left(R S\left(Z^{+}\right), A\right) \rightarrow \pi_{1}\left(S\left(Z^{+}\right), A\right)$ can be represented by a closed loop in $R S\left(Z^{+}\right)$which in $R S(Z)$ is spanned by a triangulated 2-disc supporting a positive 1-cocycle. These cocycles are used in work of Kim-Roush that leads to a counterexample to Williams' lifting problem for automorphisms of finite subsystems of subshifts of finite type.
\end{abstract}

\section{INTRODUCTION}

R. F. Williams [Wi] introduced shift equivalence SE and strong shift equivalence SSE over the non-negative integers $Z^{+}$in connection with the classification of subshifts of finite type $\left(X_{A}, \sigma_{A}\right)$ up to topological conjugacy, and it remains an open question whether SE and SSE are the same over the nonnegative integers. He did prove that SE and SSE are the same over the ring of integers $Z$. Study of the automorphism group $\operatorname{Aut}\left(\sigma_{A}\right)$ of $\left(X_{A}, \sigma_{A}\right)$ led in [W1, W2, W3] to the introduction of the $C W$-complexes $R S(\Lambda)$ of strong shift equivalences and $S(\Lambda)$ of shift equivalences over a subset $\Lambda$ of a ring which is closed under addition and multiplication. It follows from the definitions of these spaces that the set $\pi_{0}(R S(\Lambda))$ of path components of $R S(\Lambda)$ is just the set of SSE classes over $\Lambda$ and that $\pi_{0}(S(\Lambda))$ is the set of SE classes over $\Lambda$. A step in [W3] for obtaining eventual finite-order generation for inert automorphisms is the isomorphism [W3, 1.7] between the group of automorphisms $\operatorname{Aut}\left(\sigma_{A}\right)$

Received by the editors June 1, 1989.

1980 Mathematics Subject Classification (1985 Revision). Primary 54H20, 57S99, 20F99; Secondary $60 \mathrm{~J} 10$.

Key words and phrases. Higher-dimensional shift equivalence and strong shift equivalence, positive 1 -cocycle, inert automorphism.

This research was partially supported by the NSF. 
modulo the simple ones and $\left.\pi_{1}\left(R S\left(Z^{+}\right), A\right)\right)$. As known to Boyle, Fiebig, and Krieger for several years, results of the type obtained by Kim and Roush in their paper [KR] would lead to examples of a shift commuting automorphism of the periodic points of order 6 of the full 2-shift which is not the restriction of a shift commuting automorphism of the 2-shift itself. A key insight in [KR] is how to convert certain statements over $Z$ to other appropriate ones over $Z^{+}$. In particular, a fact they use is that $\pi_{1}(R S(Z), A) \rightarrow \pi_{1}(S(Z), A)$ is a monomorphism. Kim and Roush also introduce the concept of a "positive 1-cocycle" and need to know that any inert automorphism, i.e., an element in the kernel of $\pi_{1}\left(R S\left(Z^{+}\right), A\right) \rightarrow \pi_{1}\left(S\left(Z^{+}\right), A\right)$, can be represented by a closed loop in $R S\left(Z^{+}\right)$, which in $R S(Z)$ is null-homotopic by a triangulated 2-disc supporting a positive 1-cocycle. The specific construction of such cocycles given in (2.7), (2.10), and (2.16) of this paper is used in [KR].

(1.1) Theorem. Let $\Lambda$ be a principal ideal domain. Then $R S(\Lambda) \rightarrow S(\Lambda)$ is a homotopy equivalence and $\pi_{n}(R S(\Lambda), A)=\pi_{n}(S(\Lambda), A)=0$ for $n \geq 2$.

Consider Krieger's dimension group constructed over $\Lambda$ from the stationary system $A: \Lambda^{m} \rightarrow \Lambda^{m}$. (See [BLR] and [W1].) Let $\operatorname{Aut}\left(s_{A / \Lambda}\right)$ denote the group of automorphisms of this dimension group which commute with $s_{A}$, but which do not necessarily preserve the order structure. In fact, there generally is not an order structure on the dimension group unless $\Lambda$ is ordered anyway.

(1.2) Proposition. $\pi_{1}(S(\Lambda), A)=\operatorname{Aut}\left(s_{A / \Lambda}\right)$.

The proof of (1.2) is entirely similarly to [W1, 4.18]. Simply ignore any considerations of the order structure.

\section{Proof of the Main theorem (1.1) For $\pi_{0}$ AND $\pi_{1}$}

Step 1. $\left.\pi_{0}(R S(\Lambda), A)\right) \rightarrow \pi_{0}(S(\Lambda, A))$ is a bijection of sets.

From the definitions we know immediately that this map is surjective. Williams also showed that this map is injective (at least for $\Lambda=Z$ ). For completeness, we include a proof by showing

$$
\pi_{1}(S(\Lambda), R S(\Lambda))=0
$$

In other words, any path from $A$ to $B$ in $S(\Lambda)$ can be deformed back to a path from $A$ to $B$ in $R S(\Lambda)$ keeping endpoints fixed. Notation that will be used in the remainder of the paper is introduced in the proof of (2.1).

Any path from $A$ to $B$ to $S(\Lambda)$ is the concatenation of elementary shift equivalences $R: P \rightarrow Q$ or their inverses. So the proof reduces to verifying (2.1) for $R: P \rightarrow Q$. We now show how to reduce the argument further to the case where each of $P$ and $Q$ are monomorphisms and therefore are isomorphisms when tensored by $F(\Lambda)$, the field of fractions of $\Lambda$. This implies $R: P \rightarrow Q$ is also a monomorphism and an isomorphism when tensored with $F(\Lambda)$. By definition, each vertex $M$ of $R S(\Lambda)$ and of $S(\Lambda)$ is a square $m \times m$-matrix over $\Lambda$ where $m$ can vary from vertex to vertex. View $M$ as an endomorphism of 
the free $\Lambda$-module $V$ of rank $m$ equipped with the standard basis with respect to which the endomorphism $M$ has the original matrix representation $M$. We write this as $\{V, M\}$. More generally, we let $\{V, M\}$ denote a free $\Lambda$-module of finite rank equipped with a basis and an endomorphism $M$. If $M$ comes from an $m \times m$-matrix, we always take the basis to be the standard one. Now consider the SSE

$$
(\pi, M):\{V, M\} \rightarrow\{V / \operatorname{ker} M, M\}
$$

where $\pi: V \rightarrow V / \operatorname{ker} M$ is the projection and both $M: V / \operatorname{ker} M \rightarrow V$ and $M: V / \operatorname{ker} M \rightarrow V / \operatorname{ker} M$ are the homomorphisms induced by $M$. While $V$ comes with a basis, $V / \operatorname{ker} M$ does not have a canonical one. So we will choose a basis for $V / \operatorname{ker} M$ in such a way that if $\operatorname{ker} M=0$, then the basis for $V / \operatorname{ker} M=V$ remains the same. This makes (2.2) a SSE in the category of matrices over $\Lambda$.

The subgroup of those elements in $V$ which are killed by some power of $M$ is free and finitely generated because $\Lambda$ is a $P I D$ and $V$ is free of finite rank over $\Lambda$. So the construction (2.2) may be repeated a finite number of times to produce a chain of integral SSEs to a monomorphism. Moreover, starting with the shift equivalence $R:\{V, P\} \rightarrow\{W, Q\}$ over $\Lambda$, there is the following commutative diagram in $S(\Lambda)$ :

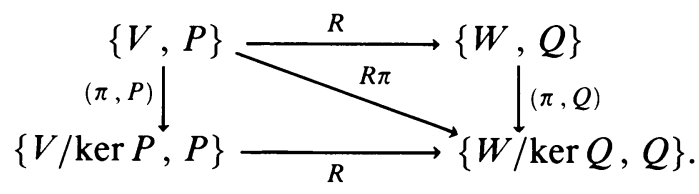

Continuing this procedure a finite number of times deforms $R:\{V, P\} \rightarrow$ $\{W, Q\}$ to a monomorphism as required.

Assume that $R:\{V, P\} \rightarrow\{W, Q\}$ is a SE where $P, Q$, and $R$ are monomorphisms. Choose $S:\{W, Q\} \rightarrow\{V, P\}$ and $k>0$ such that $R S=$ $P^{k}$ and $S R=Q^{k}$. Let $V R$ be the image of $R$ in $W$. Then we have the following diagram.

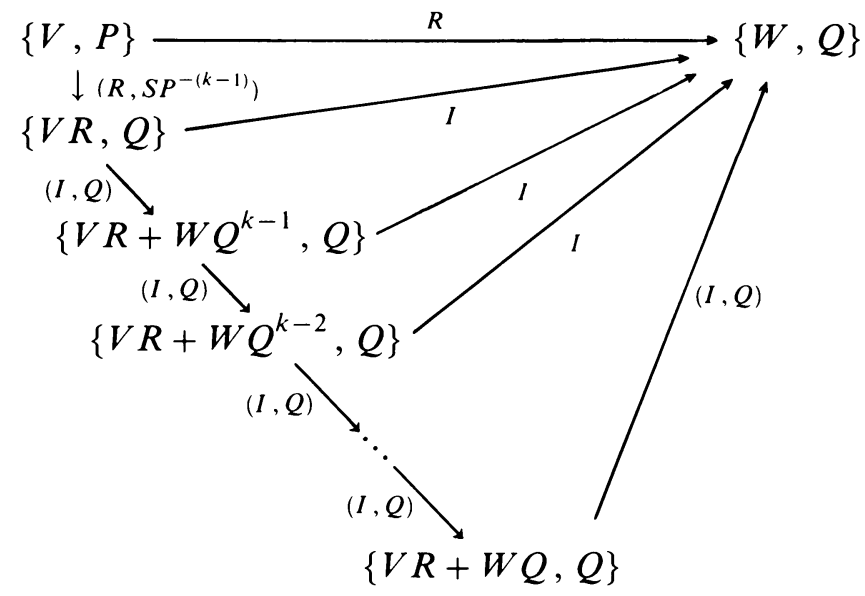


As above, we arbitrarily choose bases for the $V R+W Q^{j}$ to get a diagram in $S(\Lambda)$. This completes the proof of Step 1 .

Step 2. $\pi_{1}(R S(\Lambda), A) \rightarrow \pi_{1}(S(\Lambda), A)$ is an isomorphism.

From (2.1), we know this is surjective. Thus, we must prove it is injective. This will be done in a way which produces the "positive 1-cocycles" used by Kim-Roush in [KR].

Consider an elementary SSE $(R, S):\{V, P\} \rightarrow\{W, Q\}$. We have the diagram

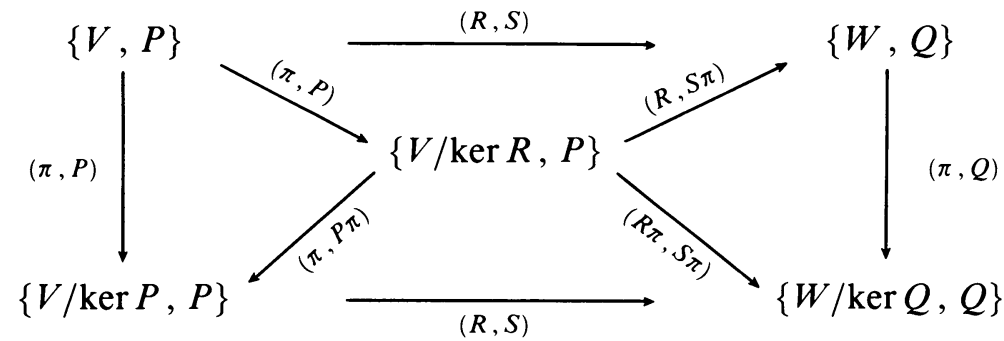

in $R S(\Lambda)$. Recall from [W2, W3] that we often let $\gamma(R, S)$ denote the path in $R S(\Lambda)$ corresponding to the elementary strong shift equivalence $(R, S): P \rightarrow$ $Q$. Moreover, we have the identities

$$
\begin{aligned}
& \gamma(R, S) \gamma(S, R)=\gamma(P, 1), \\
& \gamma(S, R) \gamma(R, S)=\gamma(Q, 1),
\end{aligned}
$$

and

$$
\gamma(1, P)=\gamma(1, Q)=1 .
$$

Using these, we can represent any element of $\pi_{1}(R S(\Lambda), A)$ as a loop

$$
V=V_{0} \rightarrow V_{1} \rightarrow \cdots \rightarrow V_{n} \leftarrow \cdots \leftarrow V_{2 n-1} \leftarrow V_{2 n}=V,
$$

where the number of forward and backward arrows is the same. Each free $\Lambda$ module $V_{i}$ comes equipped with an endomorphism $A_{i}$ and a basis giving a matrix representation for $A_{i}$. For example, if $\Lambda=Z^{+}$and the loop represents an element of $\operatorname{Aut}\left(\sigma_{A}\right)$, then the matrices are non-negative. For simplicity we have omitted the notation for the $A_{i}$, as well as for the $R$ and $S$ matrices giving the SSEs between the vertices in the path. Applying the procedure in (2.5) a finite number of times produces a homotopy of the loop (2.6) like the following $(n=2)$ :

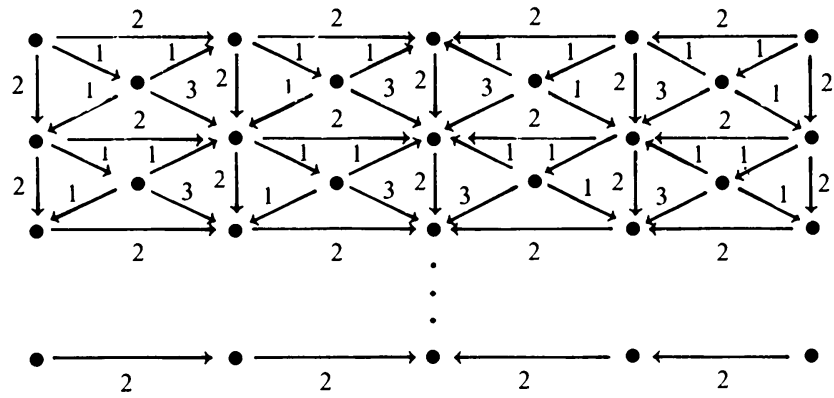


where the vertex endomorphisms and the homomorphisms in the elementary SSEs are all monomorphisms along the bottom loop. Clearly, the construction applies to all path lengths $2 n$. The right and left vertical paths are identified to obtain a homotopy of closed loops. The numbers along each edge give a specific positive 1-cocycle on the homotopy.

This notion was introduced by Kim and Roush. It consists of a function $f$ from the oriented edges of the homotopy to the positive integers satisfying the cocycle condition $f(z)=f(x)+f(y)$ whenever $x, y$, and $z$ form the boundary of a oriented triangle as in the diagram

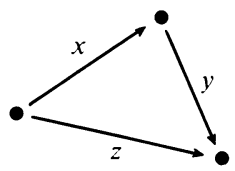

Now we assume the vertex $A$ matrices and the edge $(R, S)$ matrix pairs in the loop (2.6) are monomorphisms and we must show that when (2.6) is inert, it is possible to deform it to a point by a homotopy supporting a positive 1-cocycle fitting together with the one in (2.7). For the remainder of this section all endomorphisms and homomorphisms will be assumed to be monomorphisms.

Fix a monomorphism $A: V \rightarrow V$ of the free $\Lambda$-module $V$. As noted earlier, let $F(\Lambda)$ denote the field of fractions of $\Lambda$. Let Lat $(A)$ denote the set of lattices in $V \otimes F(\Lambda)$ which are invariant under $A$. Make $\operatorname{Lat}(A)$ into a simplicial complex by letting an $n$-simplex be an $(n+1)$-tuple $\left[L_{0}, \ldots, L_{n}\right]$ where each $L_{i}$ is a lattice in $V \otimes F(\Lambda)$ such that $L_{i} \subset L_{j}$ and $A\left(L_{j}\right) \subset L_{i}$ whenever $i<j$.

\section{(2.8) Proposition. Each connected component of $\operatorname{Lat}(A)$ is contractible.}

First, we prove that each component is simply connected. We use the notation $K \rightarrow L$ to mean $[K, L]$ is an edge in $\operatorname{Lat}(A)$. Similarly for triangles. We give the argument for the connected component containing $V$. The argument for other components is the same. So start with a loop

$$
V=L_{0} \rightarrow L_{1} \rightarrow \cdots \rightarrow L_{n} \leftarrow \cdots \leftarrow L_{2 n-1} \leftarrow L_{2 n}=V
$$

in $\operatorname{Lat}(A)$. The well-known formula for the product of a matrix and its classical adjoint implies that the intersection of two lattices in $V \otimes F(\Lambda)$ is again a lattice. Apply this to produce the null-homotopy of (2.9) in $\operatorname{Lat}(A)$ when $n=2$ as in diagram (2.10) below.

The numbers along the edges give a positive 1-cocycle. The construction clearly generalizes to any path length $2 n$. The two 45-degree paths arising from the lowest vertex are identified to obtain a homotopy of closed loops. The diagram in (2.10) resembles the one in the proof of [BFK, 2.12]. 
$(2.10)$

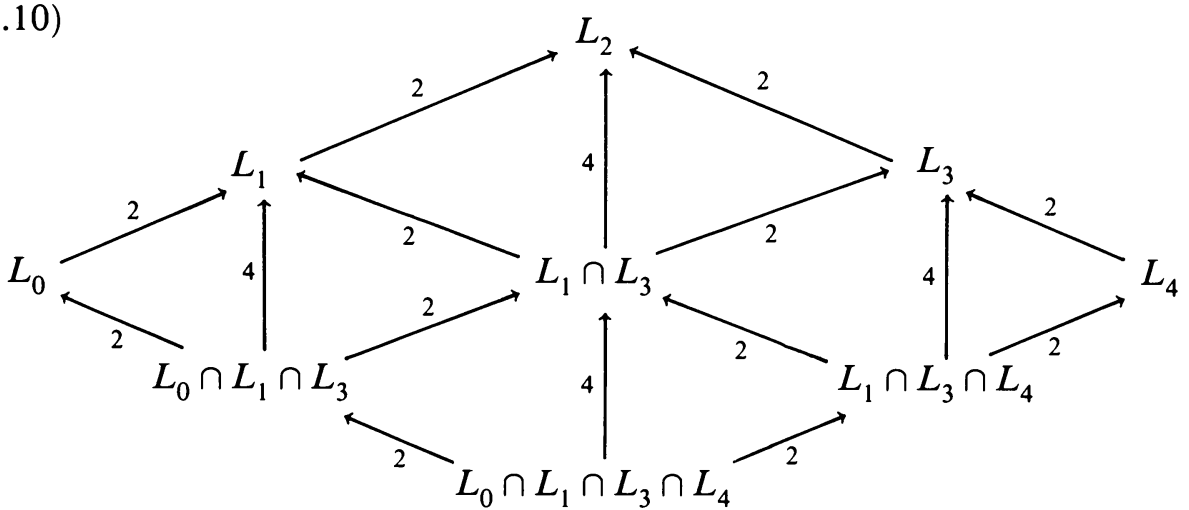

To finish the proof of (2.8), we must show that the higher homotopy groups of each component of $\operatorname{Lat}(A)$ vanish. By the Whitehead Theorem [Sp], it suffices to show that the higher homology groups are zero, because we have just shown that the components are simply connected. The proof for this is essentially a verbatim copy of the proof for Step III in [W1, 2.12]. The notation $U \rightarrow V$ for Markov partitions is replaced by the notation $K \rightarrow L$ for lattices.

Finally, we can finish the proof of Step 2 by fitting $(2.7)$ and (2.10) together via (2.16) below. Represent an inert element $\alpha$ of $\pi_{1}(R S(\Lambda), A)$ by a loop

$$
\alpha=\prod_{i=1}^{2 n} \gamma\left(R_{i}, S_{i}\right)^{\varepsilon_{i}}
$$

where $\left(R_{i}, S_{i}\right):\left(V_{i-1}, A_{i-1}\right) \rightarrow\left(V_{i}, A_{i}\right)$ and $\varepsilon_{i}=+1$ for $1 \leq i \leq n$, and $\left(R_{i}, S_{i}\right):\left(V_{i}, A_{i}\right) \rightarrow\left(V_{i-1}, A_{i-1}\right)$ and $\varepsilon_{i}=-1$ for $n+1 \leq i \leq 2 n$. For $1 \leq i \leq 2 n$, let $B_{i}$ denote the isomorphism from $V \otimes F(\Lambda)=V_{0} \otimes F(\Lambda)$ to $V_{i} \otimes F(\Lambda)$ given by the formula

$$
B_{i}=\prod_{p=1}^{i}\left(R_{p} \otimes 1\right)^{\varepsilon_{p}} .
$$

Let $B_{0}=I$. Observe that since $\alpha$ is inert, we also have $B_{2 n}=I$. Moreover, we have $A=B_{i} A_{i} B_{i}^{-1}$ for each $i$. Let $W_{i}$ be the lattice in $V \otimes F(\Lambda)$ defined by the equation

$$
W_{i} B_{i}=V_{i} .
$$

Then we have the following two diagrams in $R S(\Lambda)$ for the two cases $\varepsilon_{i}=+1$ and $\varepsilon_{i}=-1$, respectively.

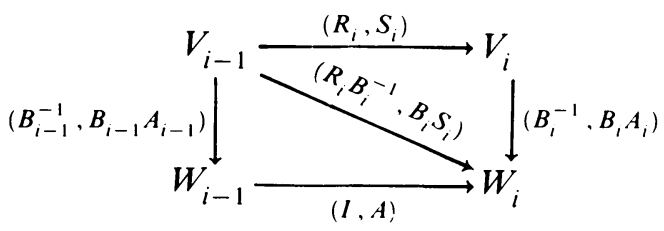




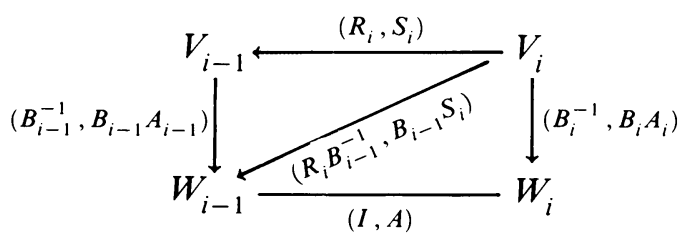

Diagrams (2.14) and (2.15) piece together to form the homotopy in (2.16) below from the bottom line in (2.7) to the top line in (2.10). Again, we take $2 n=4$ for simplicity. The construction clearly generalizes to all lengths $2 n$.

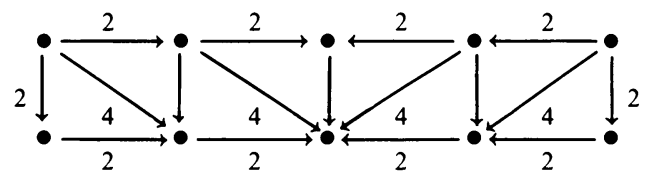

The positive 1-cocycle compatible with those in (2.7) and (2.10) is marked on the diagram. This completes the proof of Step 2.

\section{Proof of THeORem (1.1) FOR $\pi_{n}$ When $n \geq 2$}

Let $M R S(\Lambda)$ and $M S(\Lambda)$ denote the subspaces of $R S(\Lambda)$ and $S(\Lambda)$, respectively, where each SSE or SE is a monomorphism. Using generalizations of (2.3) and (2.5), we first show

(3.1) Theorem. Assume $\Lambda$ is a principal ideal domain. Then the inclusions $M S(\Lambda) \subset S(\Lambda)$ and $M R S(\Lambda) \subset R S(\Lambda)$ are homotopy equivalences.

Proof that $M S(\Lambda) \subset S(\Lambda)$ is a homotopy equivalence.

Let $\Delta=\left[V_{0}, \ldots, V_{n}\right]$ be an $n$-simplex in $S(\Lambda)$, where each $V_{i}$ is a free, based $\Lambda$-module of finite rank with an endomorphism $A_{i}$ such that whenever $i<j$, we are also given a shift equivalence $R_{i j}: V_{i} \rightarrow V_{j}$ between $A_{i}$ and $A_{j}$ satisfying $R_{i j} R_{j k}=R_{i k}$ for $i<j<k$. As in (2.2) and (2.3), let $\Delta^{\prime}=$ $\left[V_{0} / \operatorname{ker} A_{0}, \ldots, V_{n} / \operatorname{ker} A_{n}\right]$ with the corresponding vertex endomorphisms $A_{i}$ and edge shift equivalences $R_{i j}$ induced from those in $\Delta$. Let $I$ denote the unit interval. Then, as in [S], we can triangulate $\Delta \times I$ as follows: The vertices consist of the vertices $V_{i}$ of $\Delta$ together with the vertices $V_{i} / \operatorname{ker} A_{i}$ of $\Delta^{\prime}$. The directed edges are of the form $R_{i j}: V_{i} \rightarrow V_{j}, R_{i j}: V_{i} / \operatorname{ker} A_{i} \rightarrow V_{j} / \operatorname{ker} A_{j}$, $R_{i j}: V_{i} \rightarrow V_{j} / \operatorname{ker} A_{j}$ when $i<j$, and $V_{i} \rightarrow V_{i} / \operatorname{ker} A_{i}$. These satisfy the triangle relation $R_{i j} R_{j k}=R_{i k}$ for $i<j<k$, and the procedure gives

a deformation of $\Delta$ to $\Delta^{\prime}$ compatible with the face and degeneracy maps in the complex $S(\Lambda)$.

Consider a map of a pair $(X, Y)$ of finite $C W$-complexes into the pair $(S(\Lambda)$, $M S(\Lambda))$. Deform this to a cellular map so that the image of $X$ is contained in a finite subcomplex of $S(\Lambda)$. Since $\Lambda$ is a principal ideal domain, we can use the deformation (3.2) a finite number of times to deform the map on $X$ down into $M S(\Lambda)$ keeping it fixed on $Y$. 
Proof that $M R S(\Lambda) \subset R S(\Lambda)$ is a homotopy equivalence.

This proof proceeds exactly like the argument for the previous proof except that, just as (2.5) is more complicated than (2.3), so is the triangulation of $\Delta \times I$.

Let $\Delta=\left[V_{0}, \ldots, V_{n}\right]$ be an $n$-simplex in $R S(\Lambda)$ where each $V_{i}$ is a free, based $\Lambda$-module of finite rank equipped with an endomorphism $A_{i}$ and such that whenever $i<j$, we are also given a strong shift equivalence $\left(R_{i j}, S_{j i}\right)$ : $V_{i} \rightarrow V_{j}$ between $A_{i}$ and $A_{j}$ satisfying the Triangle Identities in [W3] for $i<j<k$. As in (2.2) and (2.5), let $\Delta^{\prime}=\left[V_{0} / \operatorname{ker} A_{0}, \ldots, V_{n} / \operatorname{ker} A_{n}\right]$ with the corresponding vertex endomorphisms $A_{i}$ and edge strong shift equivalences $\left(R_{i j}, S_{j i}\right)$ induced from those in $\Delta$. We will define a triangulation of $\Delta \times I$ which will be isomorphic to the cone from an interior "center" vertex $C$ of $\Delta \times I$ to an inductively defined triangulation of $\partial(\Delta \times I)$. For any simplex $\Delta$, let $C=V_{0} / \operatorname{ker} R_{01}$ equipped with the induced endomorphism $A_{0}$. The vertices of $\Delta \times I$ will be of four types:

(a) the $V_{i}$ of $\Delta$,

(b) the $V_{i} / \operatorname{ker} A_{i}$ of $\Delta^{\prime}$,

(c) the centers $C_{i}$ of the faces $\left(\partial_{i} \Delta\right) \times I$ for $0 \leq i \leq n$, and

(d) the center $C$ of $\Delta \times I$.

From the definition of the centers, we see that $C_{i}$ is given by

$$
C_{i}= \begin{cases}V_{1} / \operatorname{ker} R_{12}, & \text { for } i=0 \\ V_{0} / \operatorname{ker} R_{02}, & \text { for } i=1 \\ V_{0} / \operatorname{ker} R_{01}, & \text { for } i \geq 2\end{cases}
$$

The edges are

(ea) $\left(R_{i j}, S_{j i}\right): V_{i} \rightarrow V_{j}$ in $\Delta$,

(eb) $\left(R_{i j}, S_{j i}\right): V_{i} / \operatorname{ker} A_{i} \rightarrow V_{j} / \operatorname{ker} A_{j}$ in $\Delta^{\prime}$,

(ec) the edges in $\left(\partial_{i} \Delta\right) \times I$, and

(ed) a directed edge connecting $C$ and $V$ for each vertex $V$ of the three types (a), (b), and (c).

More specifically, in the situation (ed) we have the following cases.

$V$ is of Type (a):

If $V=V_{0}$ in $\Delta$, then $V \rightarrow C$ is

$$
\left(\pi, A_{0}\right): V_{0} \rightarrow V_{0} / \operatorname{ker} R_{01} .
$$

If $V=V_{i}$ in $\Delta$ for $i \geq 1$, then $C \rightarrow V$ is

$$
\left(R_{0 i}, S_{i 0} \pi\right): V_{0} / \operatorname{ker} R_{01} \rightarrow V_{i} .
$$

$V$ is of Type (b):

If $V=V_{i} / \operatorname{ker} A_{i}$ in $\Delta^{\prime}$ for $i \geq 0$, then $C \rightarrow V$ is

$$
\begin{array}{cc}
\left(\pi, A_{0}\right): V_{0} / \operatorname{ker} R_{01} \rightarrow V_{0} / \operatorname{ker} A_{0} \quad \text { for } i=0, \text { and } \\
\left(R_{0 i}, S_{i 0}\right): V_{0} / \operatorname{ker} R_{01} \rightarrow V_{i} / \operatorname{ker} A_{i} \quad \text { for } i \geq 1 .
\end{array}
$$


$V$ is of Type (c):

If $V=C_{i}$, then $C \rightarrow V$ is

$$
\begin{gathered}
\left(R_{01}, S_{10}\right): V_{0} / \operatorname{ker} R_{01} \rightarrow V_{1} / \operatorname{ker} R_{12}, \text { for } i=0, \\
\left(\pi, A_{0}\right): V_{0} / \operatorname{ker} R_{01} \rightarrow V_{0} / \operatorname{ker} R_{02}, \text { for } i=1, \text { and } \\
\left(I, A_{0}\right): V_{0} / \operatorname{ker} R_{01} \rightarrow V_{0} / \operatorname{ker} R_{01}, \text { for } i \geq 2 .
\end{gathered}
$$

The Triangle Identities are satisfied by any triple of edges which could possibly form a triangle, and hence we get a triangulation of $\Delta \times I$ in $R S(\Lambda)$. This gives

a deformation of $\Delta$ to $\Delta^{\prime}$ compatible with the face and degeneracy maps in the complex $R S(\Lambda)$.

Now we will proceed in the same manner to complete the proof of (3.1).

Proof that $\pi_{n}(\operatorname{MRS}(\Lambda), A)=0$ when $n \geq 2$.

This is very similar to $\S 4$ of [W2]. Let $M R S(\Lambda)_{A}$ denote the component of $M R S(\Lambda)$ containing $A$. The universal cover $\widetilde{M R S}(\Lambda)_{A}$ of $M R S(\Lambda)_{A}$ is the realization of the following simplicial set. The $n$-simplices are pairs $(\gamma, \Delta)$ where

$\Delta$ is an $n$-simplex of $\widetilde{M R S}(\Lambda)$ given by the data $\left[V_{0}, \ldots, V_{n}\right]$ and $\left(R_{i j}, S_{j i}\right): V_{i} \rightarrow V_{j}$, and $\gamma$ is a homotopy class of paths from $V$ to $V_{0}$.

The $i$ th face operator acts on $\Delta$ just as it does in $M R S(\Lambda)_{A}$. For $1 \leq i \leq n$, it leaves $\gamma$ unchanged, and for $i=0$, it changes $\gamma$ to $\gamma * \gamma\left(R_{01}, S_{10}\right)$. The covering map

$$
\widetilde{M R S}(\Lambda)_{A} \rightarrow \operatorname{MRS}(\Lambda)_{A}
$$

is induced by the map of simplicial sets taking $(\gamma, \Delta)$ to $\Delta$.

Let $\gamma$ be a path from $V$ to $V^{\prime}$ in $M R S(\Lambda)_{A}$ written in the form

$$
\gamma=\prod_{i=1}^{n} \gamma\left(R_{i}, S_{i}\right)^{\varepsilon_{i}},
$$

where $\left(R_{i}, S_{i}\right):\left\{V_{i-1}, A_{i-1}\right\} \rightarrow\left\{V_{i}, A_{i}\right\}$ when $\varepsilon_{i}=+1$ and $\left(R_{i}, S_{i}\right):\left\{V_{i}, A_{i}\right\}$ $\rightarrow\left\{V_{i-1}, A_{i-1}\right\}$ when $\varepsilon_{i}=-1$. Let $\Theta(\gamma)$ denote the isomorphism from $V \otimes$ $F(\Lambda)$ to $V^{\prime} \otimes F(\Lambda)$ given by the formula

$$
\Theta(\gamma)=\prod_{i=1}^{n}\left(R_{i} \otimes 1\right)^{\varepsilon_{i}} .
$$

For each $n$-simplex $(\gamma, \Delta)$ of $\widetilde{M R} S(\Lambda)_{A}$, let $L_{i}$ be the lattice in $V \otimes F(\Lambda)$ that is the image of $V_{i}$ under $\Theta\left(\gamma * \gamma\left(R_{0 i}, S_{i 0}\right)\right)^{-1}$. Then $\left[L_{0}, \ldots, L_{n}\right]$ is an $n$-simplex in $\operatorname{Lat}(A)$, and the correspondence taking $(\gamma, \Delta)$ to $\left[L_{0}, \ldots, L_{n}\right]$ is a map of simplicial sets. This gives a continuous map

$$
\widetilde{M R S}(\Lambda)_{A} \rightarrow \operatorname{Lat}(A) \text {. }
$$


Let $K$ and $L$ be $A$-invariant lattices in $V \otimes F(\Lambda)$ such that $A(L) \subset K$. This gives rise to a path $(I, A): K \rightarrow L$ in $M R S(\Lambda)_{A}$, and the correspondence preserves the Triangle Identities. Therefore, there is a continuous map

$$
\operatorname{Lat}(A) \rightarrow M R S(\Lambda)_{A} .
$$

Moreover, it follows from [S] and from conjugation squares like (2.14) and (2.15) that there is a homotopy commutative diagram

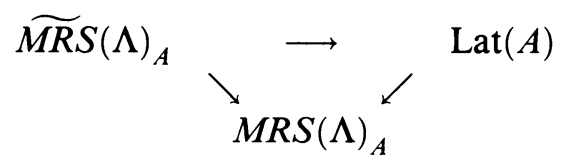

Since the components of $\operatorname{Lat}(A)$ are contractible, we see that the homomorphism from $\pi_{n}\left(\widetilde{M R S}(\Lambda)_{A}, A\right)$ to $\pi_{n}\left(M R S(\Lambda)_{A}, A\right)$ is zero. On the other hand, it is an isomorphism for $n \geq 2$, because $\widetilde{M R S}(\Lambda)_{A}$ is the universal cover.

Proof that $\pi_{n}(M S(\Lambda), A)=0$ when $n \geq 2$.

This proof follows just like the argument for $M R S(\Lambda)$, except that the definition of $\operatorname{Lat}(A)$ is changed slightly. Namely, an $n$-simplex is an $(n+1)$-tuple $\left[L_{0}, \ldots, L_{n}\right]$ where each $L_{i}$ is an $A$-invariant lattice in $V \otimes F(\Lambda)$ such that $L_{i} \subset L_{j}$, and there is a positive integer $k$ so that $A^{k}\left(L_{j}\right) \subset L_{i}$ whenever $i<j$.

\section{REFERENCES}

[BFK] M. Boyle, J. Franks, and B. Kitchens, Automorphisms of one-sided subshifts of finite type, preprint, IBM Research, T. J. Watson Research Center, Yorktown Heights, 1988. (To appear in Ergodic Theory and Dynamical Systems.)

[BLR] M. Boyle, D. Lind, and D. Rudolph, The automorphism group of a subshift of finite type, preprint, University of Washington/University of Maryland, 1986. (To appear in Trans. Amer. Math. Soc.)

[KR] K. H. Kim and F. W. Roush, On the structure of inert automorphisms of subshifts, preprint, Alabama State University, 1989.

[S] G. Segal, Classifying spaces and spectral sequences, Publ. Math. IHES. 34 (1968), 85-147.

[Sp] E. Spanier, Algebraic topology, McGraw-Hill, 1966.

[W1] J. B. Wagoner, Markov partitions and $K_{2}$, Publ. Math. IHES. 65 (1987), 91-129.

[W2] _ Triangle identities and symmetries of a subshift of finite type, preprint, Univ. of California at Berkeley, 1987. (To appear in Pacific J. Math.)

[W3] __ Eventual finite-order generation for the kernel of the dimension group representation, preprint, Univ. of California at Berkeley, 1987. (To appear in Trans. Amer. Math. Soc.)

[Wi] R. F. Williams, Classification of subshifts of finite type, Ann. of Math. 98 (1973), 120-153, Errata, ibid., 99 (1974), 380-381.

Department of Mathematics, University of California, Berkeley, California 94720 